\title{
AN EXTENSION OF PANJER'S RECURSION
}

\author{
BY \\ Klaus Th. Hess, Anett Liewald and Klaus D. Schmidt
}

Lehrstuhl für Versicherungsmathematik

Technische Universität Dresden

\begin{abstract}
Sundt and Jewell have shown that a nondegenerate claim number distribution $Q=\left\{q_{n}\right\}_{n \in \mathbf{N}_{0}}$ satisfies the recursion

$$
q_{n+1}=\left(a+\frac{b}{n+1}\right) q_{n}
$$

for all $n \geq 0$ if and only if $Q$ is a binomial, Poisson or negativebinomial distribution. This recursion is of interest since it yields a recursion for the aggregate claims distribution in the collective model of risk theory when the claim size distribution is integer-valued as well. A similar characterization of claim number distributions satisfying the above recursion for all $n \geq 1$ has been obtained by Willmot. In the present paper we extend these results and the subsequent recursion for the aggregate claims distribution to the case where the recursion holds for all $n \geq k$ with arbitrary $k$. Our results are of interest in catastrophe excess-of-loss reinsurance.
\end{abstract}

\section{INTRODUCTION}

A claim number distribution is a sequence $Q=\left\{q_{n}\right\}_{n \in \mathbf{N}_{0}}$ satisfying $q_{n} \geq 0$ for all $n \in \mathbf{N}_{0}:=\{0,1, \ldots\}$ and $\sum_{n=0}^{\infty} q_{n}=1$. A claim number distribution $\left\{q_{n}\right\}_{n \in \mathbf{N}_{0}}$ is said to be nondegenerate if $q_{n}<1$ holds for all $n \in \mathbf{N}_{0}$.

A nondegenerate claim number distribution $Q=\left\{q_{n}\right\}_{n \in \mathbf{N}_{0}}$ is said to be the Panjer distribution with parameters $a, b \in \mathbf{R}$ and $k \in \mathbf{N}_{0}$ if $q_{n}=0$ for all $n \leq$ $k-1$ and

$$
q_{n+1}=\left(a+\frac{b}{n+1}\right) q_{n}
$$

for all $n \geq k$; in this case we write $Q=$ Panjer $(a, b ; k)$. The Panjer distribution Panjer $(a, b ; k)$ is also said to be a Panjer distribution of order $k$ and the collection of all Panjer distributions of order $k$ is called the Panjer class of order $k$. 
Sundt and Jewell (1981) have shown that the Panjer class of order 0 is identical with the collection of all (nondegenerate) binomial, Poisson, or negativebinomial distributions, and Willmot (1988) has identified all distributions of the Panjer class of order 1 . In the present paper, we identify all distributions of the Panjer class of order $k$ with arbitrary $k$ (Section 3 ).

The Panjer class of order 0 is important since Panjer (1981) has shown that, in the collective model of risk theory, the aggregate claims distribution can be computed by recursion when the claim number distribution is a Panjer distribution of order 0 and the claim size distribution is concentrated on the nonnegative integers (and hence is a claim number distribution itself). In the present paper, we also obtain an extension of Panjer's recursion to the Panjer class of order $k$ with arbitrary $k$ (Section 4$)$.

The proofs of these results rely on a differential equation which characterizes the probability generating function of a Panjer distribution (Section 2).

To complete the discussion of Panjer distributions and their compound distributions, we also present some results on their binomial moments which imply that every distribution of a Hofmann family has finite moments of any order (Section 5).

Let us finally note that Panjer distributions of order $k \geq 1$ are suitable for portfolios of risks which are subject to catastrophe excess-of-loss reinsurance where, as a rule, the priority is exceeded only when at least $k$ claims occur.

\section{A DifFERENTIAL EQUATION}

In the present section we characterize the Panjer distribution Panjer $(a, b ; k)$ by a differential equation for its probability generating function. This result will be used to identify all distributions of the Panjer class of order $k$ and to extend Panjer's recursion for the aggregate claims distribution in the collective model of risk theory.

For a claim number distribution $Q=\left\{q_{n}\right\}_{n \in \mathbf{N}_{0}}$, the probability generating function $m_{Q}:[0,1] \rightarrow[0,1]$ is defined by

$$
m_{Q}(t):=\sum_{n=0}^{\infty} q_{n} t^{n}
$$

Then we have $q_{n}=m_{Q}^{(n)}(0) / n$ ! for all $n \in \mathbf{N}_{0}$.

2.1. Theorem. Let $Q=\left\{q_{n}\right\}_{n \in \mathrm{N}_{0}}$ be a nondegenerate claim number distribution. For $a, b \in \mathbf{R}$ and $k \in \mathbf{N}_{0}$, the following are equivalent:

(a) $Q=$ Panjer $(a, b ; k)$

(b) For every $l \geq 1, m_{Q}$ satisfies the differential equation

$$
(1-a t) h^{(t)}(t)=(l a+b) h^{(l-1)}(t)+q_{k}\left(\begin{array}{l}
k \\
l
\end{array}\right) l ! t^{k-l}
$$

with $t \in[0,1)$ and the initial conditions $h^{(j)}(0)=0$ for all $j \leq k-1$. 
(c) $m_{Q}$ satisfies the differential equation

$$
(1-a t) h^{(k+1)}(t)=((k+1) a+b) h^{(k)}(t)
$$

with $t \in[0,1)$ and the initial conditions $h^{(j)}(0)=0$ for all $j \leq k-1$.

Proof. Assume first that (a) holds. Then we have

$$
\begin{aligned}
\frac{m_{Q}^{(l)}(t)}{l !}-q_{k}\left(\begin{array}{c}
k \\
l
\end{array}\right) t^{k-l} & =\sum_{n=k+1}^{\infty} q_{n}\left(\begin{array}{l}
n \\
l
\end{array}\right) t^{n-l} \\
& =\sum_{n=k+1}^{\infty}\left(a+\frac{b}{n}\right) q_{n-1}\left(\begin{array}{l}
n \\
l
\end{array}\right) t^{n-l} \\
& =\sum_{j=k}^{\infty}\left(a+\frac{b}{j+1}\right) q_{j}\left(\begin{array}{c}
j+1 \\
l
\end{array}\right) t^{j+1-l} \\
& =a t \sum_{j=k}^{\infty} q_{j}\left(\begin{array}{l}
j \\
l
\end{array}\right) t^{j-l}+\left(a+\frac{b}{l}\right) \sum_{j=k}^{\infty} q_{j}\left(\begin{array}{c}
j \\
l-1
\end{array}\right) t^{j-(l-1)} \\
& =a t \frac{m_{Q}^{(l)}(t)}{l !}+\left(a+\frac{b}{l}\right) \frac{m_{Q}^{(l-1)}(t)}{(l-1) !}
\end{aligned}
$$

and hence

$$
(1-a t) m_{Q}^{(t)}(t)=(l a+b) m_{Q}^{(i-1)}(t)+q_{k}\left(\begin{array}{l}
k \\
l
\end{array}\right) l ! t^{k-l} .
$$

Therefore, (a) implies (b). Obviously, (b) implies (c). Assume now that (c) holds. By induction, we obtain

$$
(1-a t) m_{Q}^{(n+1)}(t)=((n+1) a+b) m_{Q}^{(n)}(t)
$$

for all $n \geq k$. Letting $t:=0$, the previous identity yields

$$
q_{n+1}=\left(a+\frac{b}{n+1}\right) q_{n}
$$

for all $n \geq k$ and the initial conditions yield $q_{n}=0$ for all $n \leq k-1$. Therefore, (c) implies (a).

Theorem 2.1 is known in the case $k=0$; see Schmidt (2001; Satz 7.2.2).

For a claim number distribution $Q=\left\{q_{n}\right\}_{n \in \mathrm{N}_{0}}$ and $l \in \mathbf{N}_{0}$, the binomial moment of order is defined to be

$$
\beta_{Q}^{[l]}:=\sum_{n=0}^{\infty}\left(\begin{array}{l}
n \\
l
\end{array}\right) q_{n}=\sum_{n=l}^{\infty}\left(\begin{array}{l}
n \\
l
\end{array}\right) q_{n} .
$$


The binomial moment of order $l$ is finite if and only if $\lim _{t \rightarrow 1} m_{Q}^{(t)}(t)$ is finite, and in this case we have

$$
\beta_{Q}^{[l]}=\frac{m_{Q}^{(l)}(1)}{l !}
$$

Furthermore, a binomial moment is finite if and only if the (ordinary) moment of the same order is finite. The following result is immediate from Theorem 2.1:

2.2. Corollary. Assume that $Q=\operatorname{Panjer}(a, b ; k)$ with $a<1$. Then $Q$ has finite moments of any order.

\section{THE Distributions OF THE PANJer Class OF ORdER $k$}

A claim number distribution $Q=\left\{q_{n}\right\}_{n \in \mathrm{N}_{0}}$ is the

- binomial distribution $\mathbf{B}(m, \vartheta)$ with parameters $m \in\{1,2, \ldots\}$ and $\vartheta \in(0,1)$ if

$$
q_{n}=\left(\begin{array}{c}
m \\
n
\end{array}\right) \vartheta^{n}(1-\vartheta)^{m-n}
$$

holds for all $n \in \mathbf{N}_{0}$.

- Poisson distribution $\mathbf{P}(\alpha)$ with parameter $\alpha \in(0, \infty)$ if

$$
q_{n}=e^{-\alpha} \frac{\alpha^{n}}{n !}
$$

holds for all $n \in \mathbf{N}_{0}$.

- negativebinomial distribution $\mathbf{N B}(\beta, \vartheta)$ with parameters $\beta \in(0, \infty)$ and $\vartheta \in(0,1)$ if

$$
q_{n}=\left(\begin{array}{c}
\beta+n-1 \\
n
\end{array}\right)(1-\vartheta)^{\beta} \vartheta^{n}
$$

holds for all $n \in \mathbf{N}_{0}$.

- logarithmic distribution $\log (\vartheta)$ with parameter $\vartheta \in(0,1)$ if

$$
q_{n}=\frac{1}{|\log (1-\vartheta)|} \frac{\vartheta^{n}}{n}
$$

holds for all $n \geq 1$. 
- extended negativebinomial distribution $\mathbf{E N B}(m, \beta, \vartheta)$ with parameters $m \in\{1$, $2, \ldots\}, \beta \in(-m,-m+1)$ and $\vartheta \in(0,1]$ if

$$
q_{n}=\frac{\left(\begin{array}{c}
\beta+n-1 \\
n
\end{array}\right) \vartheta^{n}}{(1-\vartheta)^{-\beta}-\sum_{j=0}^{m-1}\left(\begin{array}{c}
\beta+j-1 \\
j
\end{array}\right) \vartheta^{j}}
$$

holds for all $n \geq m$.

- extended logarithmic distribution $\mathbf{E L o g}(m, \vartheta)$ with parameters $m \in\{2,3, \ldots\}$ and $\vartheta \in(0,1]$ if

$$
q_{n}=\frac{\left(\begin{array}{c}
n \\
m
\end{array}\right)^{-1} \vartheta^{n}}{\sum_{j=m}^{\infty}\left(\begin{array}{c}
j \\
m
\end{array}\right)^{-1} \vartheta^{j}}
$$

holds for all $n \geq m$.

These distributions will be refered to as basic claim number distributions.

\subsection{Remarks}

- In the ratio defining the probabilities of the extended negativebinomial distribution, the numerator is either strictly positive for all $n \geq m$ or strictly negative for all $n \geq m$ and we also have

$$
\sum_{n=0}^{\infty}\left(\begin{array}{c}
\beta+n-1 \\
n
\end{array}\right) \vartheta^{n}=\sum_{n=0}^{\infty}\left(\begin{array}{c}
-\beta \\
n
\end{array}\right)(-\vartheta)^{n}=(1-\vartheta)^{-\beta}
$$

Therefore, the extended negativebinomial distribution is well-defined.

- For $m \geq 2$, we have

$$
\sum_{n=m}^{\infty}\left(\begin{array}{c}
n \\
m
\end{array}\right)^{-1}=\sum_{j=0}^{\infty}\left(\begin{array}{c}
j+m \\
m
\end{array}\right)^{-1}=\frac{m}{m-1}
$$

Therefore, the extended logarithmic distribution is well-defined.

- Willmot (1988) used the term extended truncated negativebinomial distribution for ENB $(1, \beta, \vartheta)$; see also Klugman, Panjer and Willmot (1998) and Willmot and Lin (2001). Although there is an obvious justification for this terminology, we prefer to omit the adjective truncated here since our results suggest to reverse the order of truncation and extension and to consider 
extended negativebinomial distributions and truncated extended negativebinomial distributions.

- Apparently, the distributions $\mathbf{E N B}(m, \beta, \vartheta)$ with $m \geq 2$ and $\operatorname{ELog}(m, \vartheta)$ have not been considered before; see Johnson, Kotz and Kemp (1992).

It is easily seen that every basic claim number distribution is a Panjer distribution. Table 1 below contains for every basic claim number distribution $Q$ considered as Panjer $(a, b ; k)$ the parameters $a, b, k$ and the probability generating function $m_{Q}$ :

TABLE 1

BASIC CLAIM NUMBER DISTRIBUTIONS

\begin{tabular}{lllll}
\hline \hline $\boldsymbol{Q}$ & $\boldsymbol{a}$ & $\boldsymbol{b}$ & $\boldsymbol{k}$ & $\boldsymbol{m}_{Q}(t)$ \\
\hline $\mathbf{B}(m, \vartheta)$ & $-\frac{\vartheta}{1-\vartheta}$ & $(m+1) \frac{\vartheta}{1-\vartheta}$ & 0 & $(1-\vartheta+\vartheta t)^{m}$ \\
$\mathbf{P}(\alpha)$ & 0 & $\alpha$ & 0 & $e^{-\alpha(1-t)}$ \\
$\mathbf{N B}(\beta, \vartheta)$ & $\vartheta$ & $(\beta-1) \vartheta$ & 0 & $\left(\frac{1-\vartheta t}{1-\vartheta}\right)^{-\beta}$ \\
$\mathbf{L o g}(\vartheta)$ & $\vartheta$ & $-\vartheta$ & 1 & $\frac{\log (1-\vartheta t)}{\log (1-\vartheta)}$ \\
$\mathbf{E N B}(m, \beta, \vartheta)$ & $\vartheta$ & $(\beta-1) \vartheta$ & $m$ & $\frac{(1-\vartheta t)^{-\beta}-\sum_{j=0}^{m-1}\left(\frac{\beta+j-1}{j}\right)(\vartheta t)^{j}}{(1-\vartheta)^{-\beta}-\sum_{j=0}^{m-1}\left(\frac{\beta+j-1}{j}\right) \vartheta^{j}}$ \\
$\mathbf{E L o g}(m, \vartheta)$ & $\vartheta$ & $-m \vartheta$ & $m$ & $\frac{\sum_{n=m}^{\infty}\left(\begin{array}{l}n \\
m\end{array}\right)^{-1}(\vartheta t)^{n}}{\sum_{n=m}^{\infty}\left(\begin{array}{l}n \\
m\end{array}\right)^{-1} \vartheta^{n}}$ \\
\hline \hline
\end{tabular}

\subsection{Remarks}

- Table 1 shows that there exist Panjer distributions Panjer $(a, b ; k)$ with $a=1$; this has first been observed by Willmot (1988) who discovered $\operatorname{ENB}(1, \beta, 1)$ $=$ Panjer $(1, \beta-1 ; 1)$.

- For $\mathbf{E N B}(m, \beta, 1)=$ Panjer $(1, \beta-1 ; m)$ the moment of order $l$ is finite if and only if $l \leq m-1$, and for $\operatorname{ELog}(m, 1)=\operatorname{Panjer}(1,-m ; m)$ the moment of order $l$ is finite if and only if $l \leq m-2$. This shows that Corollary 2.2 cannot be extended to the case $a=1$.

For a claim number distribution $Q=\left\{q_{n}\right\}_{n \in \mathrm{N}_{0}}$ and $k \in \mathbf{N}_{0}$ satisfying $q_{k}>0$ and $\sum_{n=k+1}^{\infty} q_{n}>0$, define

$$
Q^{\langle k\rangle}:=\left\{q_{n}^{\langle k\rangle}\right\}_{n \in \mathrm{N}_{0}}
$$


where $q_{n}^{\langle k\rangle}:=0$ for all $n \leq k-1$ and

$$
q_{n}^{\langle k\rangle}:=\frac{q_{n}}{1-\sum_{j=0}^{k-1} q_{j}}
$$

for all $n \geq k$. Then $Q^{\langle k\rangle}$ is a nondegenerate claim number distribution satisfying

$$
m_{Q^{\langle k\rangle}}(t)=\frac{m_{Q}(t)-\sum_{j=0}^{k-1} q_{j} t^{j}}{1-\sum_{j=0}^{k-1} q_{j}}
$$

The distribution $Q^{\langle k\rangle}$ is said to be the $k$-truncation of $Q$.

Table 2 below defines the notation for the $k$-truncation $Q^{\langle k\rangle}$ of the basic claim number distribution $Q$ :

TABLE 2

$k$-TRUNCATIONS OF BASIC CLAIM NUMBER DISTRIBUTIONS

\begin{tabular}{lll}
\hline \hline $\boldsymbol{Q}$ & $k$ & $\boldsymbol{Q}^{\langle k\rangle}$ \\
\hline $\mathbf{B}(m, \vartheta)$ & $\{0,1, \ldots, m-1\}$ & $\mathbf{B}(m, \vartheta ; k)$ \\
$\mathbf{P}(\alpha)$ & $\{0,1, \ldots\}$ & $\mathbf{P}(\alpha ; k)$ \\
$\mathbf{N B}(\beta, \vartheta)$ & $\{0,1, \ldots\}$ & $\mathbf{N B}(\beta, \vartheta ; k)$ \\
$\mathbf{L o g}(\vartheta)$ & $\{1,2, \ldots\}$ & $\mathbf{L o g}(\vartheta ; k)$ \\
$\mathbf{E N B}(m, \beta, \vartheta)$ & $\{m, m+1, \ldots\}$ & $\mathbf{E N B}(m, \beta, \vartheta ; k)$ \\
$\mathbf{E L o g}(m, \vartheta)$ & $\{m, m+1, \ldots\}$ & $\mathbf{E L o g}(m, \vartheta ; k)$ \\
\hline \hline
\end{tabular}

In particular, we have

$$
\begin{aligned}
\mathbf{B}(m, \vartheta) & =\mathbf{B}(m, \vartheta ; 0) \\
\mathbf{P}(\alpha) & =\mathbf{P}(\alpha ; 0) \\
\mathbf{N B}(\beta, \vartheta) & =\mathbf{N B}(\beta, \vartheta ; 0) \\
\mathbf{L o g}(\vartheta) & =\mathbf{L o g}(\vartheta ; 1) \\
\mathbf{E N B}(m, \beta, \vartheta) & =\mathbf{E N B}(m, \beta, \vartheta ; m) \\
\mathbf{E L o g}(m, \vartheta) & =\mathbf{E L o g}(m, \vartheta ; m)
\end{aligned}
$$

Since every basic claim number distribution is a Panjer distribution, it is clear that the $k$-truncation of a basic claim number distribution belongs to the Panjer class of order $k$. In order to prove that the converse of this assertion holds as well, we need the following lemma:

3.3. Lemma. Assume that $Q=$ Panjer $(a, b ; k)$. Then

$$
(k+1) a+b>0 \text {. }
$$

Moreover, $a+b \geq 0$ implies $a<1$, and $a+b<0$ implies $a \leq 1$. 
Proof. The first inequality is immediate since $Q$ is nondegenerate. Let us now assume that $a>0$ and $a+b \geq 0$. Then we have, for all $n \geq k$.

$$
q_{n+1}=\frac{n a+a+b}{n+1} q_{n} \geq \frac{n}{n+1} a q_{n}
$$

and hence

$$
q_{n+1} \geq \frac{k+1}{n+1} a^{n-k} q_{k+1} .
$$

Since the series $\sum_{n=k}^{\infty} \frac{k+1}{n+1} a^{n-k}$ diverges for $a \geq 1$, we obtain $a<1$. Let us next assume that $a>0$ and $a+b<0$. Then we have, for all $n \geq k$,

$$
q_{n+1}=\frac{(n-k) a+(k+1) a+b}{n+1} q_{n} \geq \frac{n-k}{n+1} a q_{n}
$$

and hence

$$
q_{n+1} \geq\left(\begin{array}{l}
n+1 \\
k+1
\end{array}\right)^{-1} a^{n-k} q_{k+1}
$$

Since the series $\sum_{n=k}^{\infty}\left(\begin{array}{l}n+1 \\ k+1\end{array}\right)^{-1} a^{n-k}$ diverges for $a>1$, we obtain $a \leq 1$.

We can now characterize the distributions of the Panjer class of order $k$ :

3.4. Theorem. Let $Q$ be a nondegenerate claim number distribution. For $k \in \mathrm{N}_{0}$, the following are equivalent:

(a) $Q$ belongs to the Panjer class of order $k$.

(b) $Q$ is the $k$-truncation of a basic claim number distribution.

Proof. Assume first that (a) holds and consider $Q=\left\{q_{n}\right\}_{n \in \mathrm{N}_{0}}=$ Panjer $(a, b ; k)$. By Theorem 2.1, we have

$$
\frac{d\left(\log m_{Q}^{(k)}\right)}{d t}(t)=\frac{(k+1) a+b}{1-a t}
$$

for all $t \in[0,1)$ and $m_{Q}^{(n)}(0)=0$ for all $n \leq k-1$. To solve the differential equation, we distinguish three cases depending on $a$ :

- The case $a<0$ : In this case we obtain

$$
m_{Q}^{(k)}(t)=c(1-a t)^{-(k+1+b / a)}
$$

for some $c \in \mathbf{R}$. Since 


$$
q_{n+1}=\frac{(n-k) a+(k+1) a+b}{n+1} q_{n}
$$

holds for all $n \geq k$, Lemma 3.3 yields the existence of some $m \geq k$ satisfying $q_{m+1}=0<q_{m}$. We obtain $m=(a+b) /(-a)$, and hence

$$
m_{Q}^{(k)}(t)=c(1-a t)^{m-k} .
$$

The general solution of this differential equation has the form

$$
m_{Q}(t)=\sum_{j=0}^{k-1} c_{j} t^{j}+c_{k}(1-a t)^{m}
$$

and the initial conditions together with $m_{Q}(1)=1$ yield

$$
m_{Q}(t)=\frac{\left(\frac{1}{1-a}+\frac{-a}{1-a} t\right)^{m}-\sum_{j=0}^{k-1}\left(\begin{array}{c}
m \\
j
\end{array}\right)\left(\frac{1}{1-a}\right)^{m-j}\left(\frac{-a}{1-a} t\right)^{j}}{1-\sum_{j=0}^{k-1}\left(\begin{array}{c}
m \\
j
\end{array}\right)\left(\frac{1}{1-a}\right)^{m-j}\left(\frac{-a}{1-a}\right)^{j}} .
$$

Therefore, we have $Q=\mathbf{B}((a+b) /(-a),(-a) /(1-a) ; k)$.

- The case $a=0$ : In this case, Lemma 3.3 yields $b \in(0, \infty)$ and we obtain

$$
m_{Q}^{(k)}(t)=c e^{b t}
$$

for some $c \in \mathbf{R}$. The general solution of this differential equation has the form

$$
m_{Q}(t)=\sum_{j=0}^{k-1} c_{j} t^{j}+c_{k} e^{b t}
$$

and the initial conditions together with $m_{Q}(1)=1$ yield

$$
m_{Q}(t)=\frac{e^{-b(1-t)}-\sum_{j=0}^{k-1} e^{-b t} \frac{(b t)^{j}}{j !}}{1-\sum_{j=0}^{k-1} e^{-b} \frac{b^{j}}{j !}} .
$$

Therefore, we have $Q=\mathbf{P}(b ; k)$.

- The case $a>0$ : In this case we obtain

$$
m_{Q}^{(k)}(t)=c(1-a t)^{-(k+1+b / a)}
$$


for some $c \in \mathbf{R}$. To solve this differential equation, we distinguish five cases depending on $b$ :

○ The case $b>-a$ : In this case we have $a+b \geq 0$ and Lemma 3.3 yields $a \in(0,1)$. Letting $\beta:=(a+b) / a$, we obtain $\beta \in(0, \infty)$ and

$$
m_{Q}^{(k)}(t)=c(1-a t)^{-(k+\beta)}
$$

The general solution of this differential equation has the form

$$
m_{Q}(t)=\sum_{j=0}^{k-1} c_{j} t^{j}+c_{k}(1-a t)^{-\beta}
$$

and the initial conditions together with $m_{Q}(1)=1$ yield

$$
m_{Q}(t)=\frac{\left(\frac{1-a t}{1-a}\right)^{-\beta}-\sum_{j=0}^{k-1}\left(\begin{array}{c}
\beta+j-1 \\
j
\end{array}\right)(1-a)^{\beta}(a t)^{j}}{1-\sum_{j=0}^{k-1}\left(\begin{array}{c}
\beta+j-1 \\
j
\end{array}\right)(1-a)^{\beta} a^{j}} .
$$

Therefore, we have $Q=\mathbf{N B}((a+b) / a, a ; k)$.

○ The case $b=-a$ : In this case we obtain

$$
m_{Q}^{(k)}(t)=c(1-a t)^{-k}
$$

for some $c \in \mathbf{R}$. The general solution of this differential equation has the form

$$
m_{Q}(t)=\sum_{j=0}^{k-1} c_{j} t^{j}+c_{k} \log (1-a t)
$$

and the initial conditions together with $m_{Q}(1)=1$ yield

$$
m_{Q}(t)=\frac{\frac{\log (1-a t)}{\log (1-a)}-\sum_{j=1}^{k-1} \frac{(a t)^{j}}{j}}{1-\sum_{j=1}^{k-1} \frac{a^{j}}{j}} .
$$

Therefore, we have $Q=\log (a ; k)$.

- The case $-(m+1) a<b<-m a$ with $m \in\{1,2, \ldots, k\}$ : In this case we have $a+b<0$ and Lemma 3.3 yields $a \in(0,1]$. Letting $\beta:=(a+b) / a$, we obtain $\beta \in(-m,-m+1)$. Proceeding as in the case $b>-a$, but taking into account the possibility of $a=1$, we obtain 


$$
m_{Q}(t)=\frac{(1-a t)^{-\beta}-\sum_{j=0}^{k-1}\left(\begin{array}{c}
\beta+j-1 \\
j
\end{array}\right)(a t)^{j}}{(1-a)^{-\beta}-\sum_{j=0}^{k-1}\left(\begin{array}{c}
\beta+j-1 \\
j
\end{array}\right) a^{j}} .
$$

Therefore, we have $Q=\mathbf{E N B}(m,(a+b) / a, a ; k)$.

O The case $b=-m a$ with $m \in\{2,3, \ldots, k\}$ : In this case we have $a+b<0$ and Lemma 3.3 yields $a \in(0,1]$. We obtain

$$
m_{Q}^{(k)}(t)=c(1-a t)^{m-k-1}
$$

for some $c \in \mathbf{R}$. The general solution of this differential equation has the form

$$
m_{Q}(t)=\sum_{j=0}^{k-1} c_{j} t^{j}+c_{k}(1-a t)^{m-1} \log (1-a t)
$$

and the initial conditions together with $m_{Q}(1)=1$ yield

$$
m_{Q}(t)=\frac{\sum_{n=k}^{\infty}\left(\begin{array}{c}
n \\
m
\end{array}\right)^{-1}(a t)^{j}}{\sum_{n=k}^{\infty}\left(\begin{array}{c}
n \\
m
\end{array}\right)^{-1} a^{j}} .
$$

Therefore, we have $Q=\mathbf{E} \log (m, a ; k)$.

O The case $b \leq-(k+1) a$ : This case is impossible because of Lemma 3.3. Therefore, (a) implies (b). The converse implication is obvious, as noticed before.

In the cases $k=0$ and $k=1$, Theorem 3.4 is due to Sundt and Jewell (1981) and Willmot (1988), respectively.

\section{PANJER's RECURSION}

Let $N$ be a random variable such that the distribution $Q$ of $N$ is a claim number distribution, let $\left\{X_{n}\right\}_{n \in \mathrm{N}}$ be a sequence of random variables which is i.i.d. and independent of $N$, and define

$$
S:=\sum_{n=1}^{N} X_{n}
$$

In the collective model of risk theory, $N$ is interpreted as the number of claims, $X_{n}$ is interpreted as the claim size of claim $n$ and $S$ is interpreted as the aggregate claim size of the portfolio. 
If the distribution $F$ of each $X_{n}$ is a claim number distribution, then this is also true for the distribution $F^{* k}=\left\{f_{n}^{* k}\right\}_{n \in \mathrm{N}_{0}}$ of $\sum_{n=1}^{k} X_{n}$ and for the distribution $\operatorname{Comp}(Q, F)$ of $S$; we have $m_{F^{*}}(t)=\left(m_{F}(t)\right)^{k}$ and $m_{\operatorname{Comp}(Q, F)}(t)=m_{Q}\left(m_{F}(t)\right)$. The following result extends Theorem 2.1:

4.1. Theorem. Let $Q=\left\{q_{n}\right\}_{n \in \mathrm{N}_{0}}$ be a nondegenerate claim number distribution. For $a, b \in \mathbf{R}$ and $k \in \mathbf{N}_{0}$, the following are equivalent:

(a) $Q=\operatorname{Panjer}(a, b ; k)$.

(b) For every claim number distribution $F=\left\{f_{n}\right\}_{n \in \mathrm{N}_{0}}$ with $f_{0}=0$ and for every $l \geq 1, m_{\mathrm{Comp}(Q, F)}$ satisfies the differential equation

$$
\left(1-a m_{F}(t)\right) h^{(t)}(t)=\sum_{i=1}^{l}\left(\begin{array}{l}
l \\
i
\end{array}\right)\left(a+b \frac{i}{l}\right) h^{(l-i)}(t) m_{F}^{(i)}(t)+q_{k} m_{F^{* k}}^{(t)}(t)
$$

with $t \in[0,1)$ and the initial conditions $h^{(j)}(0)=0$ for all $j \leq k-1$.

Proof. Assume first that (a) holds and let $G:=\operatorname{Comp}(Q, F)$. Then we have

and hence

$$
m_{G}(t)=m_{Q}\left(m_{F}(t)\right)
$$

$$
m_{G}^{\prime}(t)=m_{Q}^{\prime}\left(m_{F}(t)\right) m_{F}^{\prime}(t)
$$

Because of Theorem 2.1, this yields

$$
\begin{aligned}
\left(1-a m_{F}(t)\right) m_{G}^{\prime}(t) & =\left(1-a m_{F}(t)\right) m_{Q}^{\prime}\left(m_{F}(t)\right) m_{F}^{\prime}(t) \\
& =\left((a+b) m_{Q}\left(m_{F}(t)\right)+q_{k} k\left(m_{F}(t)\right)^{k-1}\right) m_{F}^{\prime}(t) \\
& =(a+b) m_{G}(t) m_{F}^{\prime}(t)+q_{k} m_{F}^{\prime * k}(t) .
\end{aligned}
$$

This is the differential equation of (b) in the case $l=1$, and the general case now follows by induction. Furthermore, Theorem 2.1 yields

$$
m_{Q}^{(j)}(0)=0
$$

for all $j \leq k-1$. Since $m_{F}(0)=f_{0}=0$, differentiation of both sides of the identity $m_{G}(t)=m_{Q}\left(m_{F}(t)\right)$ yields

$$
m_{G}^{(\hat{)})}(0)=0
$$

for all $j \leq k-1$. Therefore, (a) implies (b). Assume now that (b) holds. For the claim number distribution $F=\left\{f_{n}\right\}_{n \in \mathbf{N}_{0}}$ with $f_{1}=1$, we have $m_{F}(t)=t$ and hence $\operatorname{Comp}(Q, F)=Q$, and the differential equation becomes

$$
(1-a t) h^{(l)}(t)=(l a+b) h^{(l-1)}(t)+q_{k}\left(\begin{array}{l}
k \\
l
\end{array}\right) l ! t^{k-l} .
$$


By Theorem 2.1, this yields $Q=\operatorname{Panjer}(a, b ; k)$. Therefore, (b) implies (a).

When the distribution $F=\left\{f_{n}\right\}_{n \in \mathbf{N}_{0}}$ considered in Theorem 4.1 is obtained as an approximation of a continuous distribution, then the condition $f_{0}=0$ may be violated. For this case, we have the following variant of Theorem 4.1:

4.2. Theorem. Assume that $Q=\operatorname{Panjer}(a, b ; k)$. If $F=\left\{f_{n}\right\}_{n \in \mathrm{N}_{0}}$ is a claim number distribution and if $G=\operatorname{Comp}(Q, F)$, then $m_{G}(t)=m_{Q}\left(m_{F}(t)\right)$ and the identity

$$
\left(1-a m_{F}(t)\right) m_{G}^{(t)}(t)=\sum_{i=1}^{l}\left(\begin{array}{l}
l \\
i
\end{array}\right)\left(a+b \frac{i}{l}\right) m_{G}^{(l-i)}(t) m_{F}^{(i)}(t)+q_{k} m_{F^{*}}^{(t)}(t)
$$

holds for all $l \geq 1$.

With $t=0$, Theorem 4.2 yields the following extension of Panjer's recursion for the probabilities of a compound distribution:

4.3. Corollary. Assume that $Q=\operatorname{Panjer}(a, b ; k)$. If $F=\left\{f_{n}\right\}_{n \in \mathrm{N}_{0}}$ is a claim number distribution with $f_{0}<1$ and if $G=\left\{g_{n}\right\}_{n \in \mathbf{N}_{0}}=\operatorname{Comp}(Q, F)$, then $g_{0}=m_{Q}\left(f_{0}\right)$ and the identity

$$
g_{n}=\frac{1}{1-a f_{0}}\left(\sum_{i=1}^{n}\left(a+b \frac{i}{n}\right) g_{n-i} f_{i}+q_{k} f_{n}^{* k}\right)
$$

holds for all $n \geq 1$.

With $t=1$, Theorem 4.2 yields the following recursion for the binomial moments of a compound distribution:

4.4. Corollary. Assume that $Q=\operatorname{Panjer}(a, b ; k)$ with $a<1$. If $F$ is a claim number distribution and if $G=\operatorname{Comp}(Q, F)$, then $\beta_{G}^{[0]}=1$ and the identity

$$
\beta_{G}^{[n]}=\frac{1}{1-a}\left(\sum_{i=1}^{n}\left(a+b \frac{i}{n}\right) \beta_{G}^{[n-i]} \beta_{F}^{[i]}+q_{k} \beta_{F^{*}}^{[n]}\right)
$$

holds for all $n \geq 1$ such that $\beta_{F}^{[n]}$ is finite.

The results of this section are known in the case $k=0$; see Schmidt (2001; Abschnitt 7.3). Corollary 4.3 can also be obtained from Sundt (1992; Theorem 11); see also Sundt and Jewell (1981; Theorem 5). Corollary 4.4 is a variant of a result of DePril (1986) who obtained a recursion for the (ordinary) moments in the case $k=0$; see also Schmidt (1996; Theorem 5.4.3).

\section{An APpliCATION to Hofmann Families}

In this section, we consider a family $\left\{Q_{s}\right\}_{s \in[0, \infty)}$ of claim number distributions $Q_{s}=\left\{q_{s n}\right\}_{n \in \mathbf{N}_{0}}$. For $n \in \mathbf{N}_{0}$, define $\Pi_{n}:[0, \infty) \rightarrow[0,1]$ by 


$$
\Pi_{n}(s):=q_{s n} .
$$

The family $\left\{Q_{s}\right\}_{s \in[0, \infty)}$ is said to be the Hofmann family $\mathbf{H}(d, p, c)$ with parameters $d \in[0, \infty)$ and $p, c \in(0, \infty)$ if there exists a differentiable function $\vartheta:[0, \infty)$ $\rightarrow \mathbf{R}$ with the following properties:

$$
\begin{aligned}
& \vartheta(0)=0 \\
& \frac{d \vartheta}{d s}(s)=\frac{p}{(1+c s)^{d}} \\
& \Pi_{0}(s)=\exp (-\vartheta(s)) \\
& \Pi_{n}(s)=\frac{(-s)^{n}}{n !} \frac{d^{n} \Pi_{0}}{d s^{n}}(s)
\end{aligned}
$$

The Hofmann family was introduced by Hofmann (1955). Kestemont and Paris (1985) pointed out that every distribution of a Hofmann family can be written as

$$
Q_{s}=\operatorname{Comp}\left(P_{s}, R_{s}\right)
$$

where $P_{s}$ is a Poisson distribution and $R_{s}$ is a claim number distribution. The following theorem makes this statement more precise. Let Dirac(1) denote the claim number distribution $Q=\left\{q_{n}\right\}_{n \in \mathbf{N}_{0}}$ with $q_{1}=1$.

5.1. Theorem. Assume that $\left\{Q_{s}\right\}_{s \in[0, \infty)}=\mathbf{H}(d, p, c)$. Then

$$
Q_{s}=\operatorname{Comp}\left(\mathbf{P}(\vartheta(s)), R_{s}\right)
$$

holds with

$$
R_{s}= \begin{cases}\operatorname{Dirac}(1) & \text { if } d=0 \\ \mathbf{E N B}(1, d-1, c s /(1+c s)) & \text { if } d \in(0,1) \\ \mathbf{L o g}(c s /(1+c s)) & \text { if } d=1 \\ \mathbf{N B}(d-1, c s /(1+c s) ; 1) & \text { if } d \in(1, \infty)\end{cases}
$$

and for all $s \in[0, \infty)$. In particular, $Q_{s}$ has finite moments of any order.

Proof. By the Bernstein-Widder Theorem, there exists a probability distribution $Q$ concentrated on $(0, \infty)$ such that

$$
\Pi_{n}(s)=\int_{(0, \infty)} e^{-\lambda s} \frac{(\lambda s)^{n}}{n !} d Q(\lambda)
$$

holds for all $n \in \mathbf{N}_{0}$ and $s \in[0, \infty)$. Using this explicit formula for $\Pi_{n}(s)$, straightforward computation yields

$$
m_{Q_{s}}(t)=\exp (-\vartheta(s-s t))
$$


and we also have

$$
m_{\operatorname{Comp}\left(\mathbf{P}(\vartheta(s)), R_{s}\right)}(t)=\exp \left(-\vartheta(s)\left(1-m_{R_{s}}(t)\right)\right)
$$

Now the first assertion follows from

$$
\vartheta(s)= \begin{cases}p s & \text { if } d=0 \\ \frac{p}{c} \log (1+c s) & \text { if } d=1 \\ \frac{p}{c} \frac{(1+c s)^{1-d}-1}{1-d} & \text { if } d \in(0,1) \cup(1, \infty)\end{cases}
$$

and the second assertion follows from Corollary 4.4 and Corollary 2.2

\section{ACKNOWLEDGEMENT}

The authors are grateful to the referees for their comments on the first draft of this paper and to Sabine Münch for a discussion of the Hofmann family.

\section{REFERENCES}

DePriL, N. (1986) Moments of a class of compound distributions. Scand. Actuar. J., 117-120. Hofmann, M. (1955) Über zusammengesetzte Poisson-Prozesse und ihre Anwendungen in der Unfallversicherung. Mitt. Verein. Schweiz. Versicherungsmathematiker 55, 499-575.

Johnson, N.L., Kotz, S. and KeMP, A.W. (1992) Univariate Discrete Distributions. Wiley, New York and Chichester.

Kestemont, R.M. and Paris, J. (1985) Sur l'ajustement du nombre de sinistres. Mitt. Verein. Schweiz. Versicherungsmathematiker 85, 157-164.

Klugman, S.A., Panjer, H.H. and Willmot, G.E. (1998) Loss Models. Wiley, New York and Chichester.

PANJER, H.H. (1981) Recursive evaluation of a family of compound distributions. ASTIN Bull. $12,22-26$.

SCHMIDT, K.D. (1996) Lectures on Risk Theory. Teubner, Stuttgart.

SCHMIDT, K.D. (2001) Versicherungsmathematik. Springer, Berlin - Heidelberg - New York.

SundT, B. (1992) On some extensions of Panjer's class of counting distributions. ASTIN Bull. 22, 61-80.

SUNDT, B. and JEWELL, W.S. (1981) Further results of recursive evaluation of compound distributions. ASTIN Bull. 12, 27-39.

WILLMOT, G.E. (1988) Sundt and Jewell's family of discrete distributions. ASTIN Bull. 18, 17-29.

WILLMOT, G.E. and LiN, X.S. (2001) Lundberg Approximations for Compound Distributions with Insurance Applications. Springer, Berlin - Heidelberg - New York.

Klaus Th. Hess, Anett Liewald and Klaus D. Schmidt

Lehrstuhl für Versicherungsmathemetik

Technische Universität Dresden

D-01062 Dresden

E-mail: schmidt@math.tu-dresden.de 